\title{
COLEGIO ALIANZA FRANCESA JEAN MERMOZ, CURICó, CHILE
}

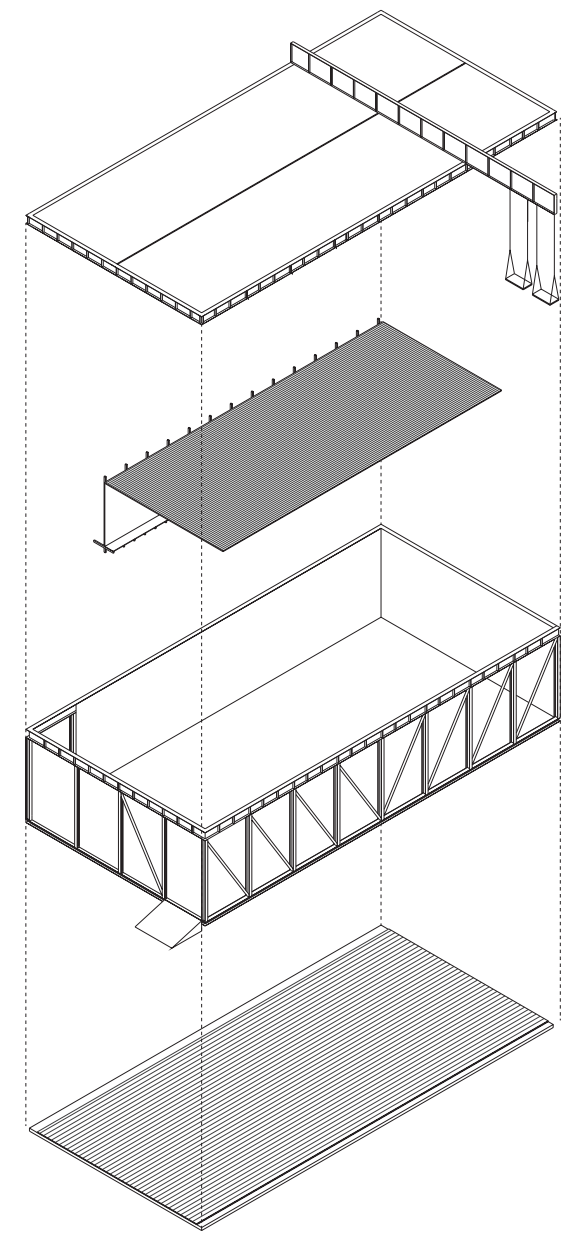

Axonométrica desplegada pabellón psicomotricidad E. 1: 500

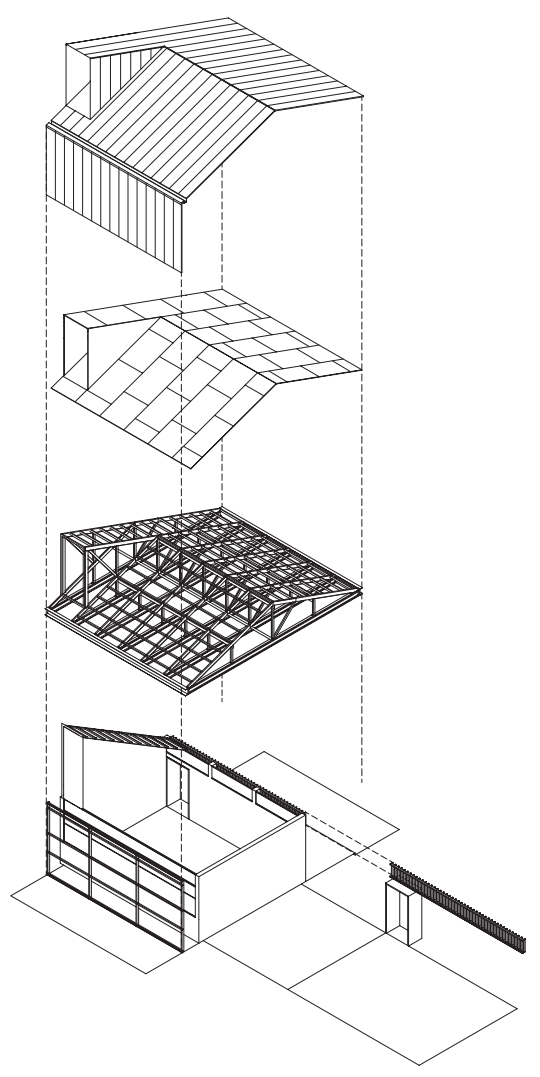

Axonométrica constructiva desplegada sala de clases E. 1: 500

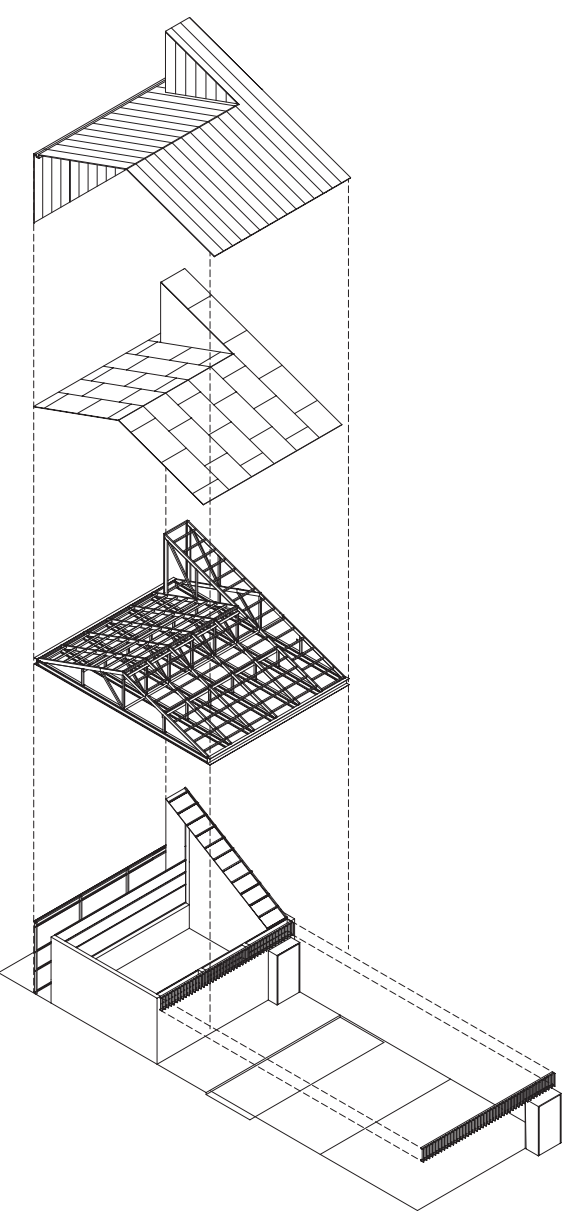

Guillermo Hevia | Profesor instructor, Pontificia Universidad Católica de Chile, Santiago

Arquitecto, Pontificia Universidad Católica de Chile, 2011 y Premio Promoción Escuela de Arquitectura Uc, 2011. En 2012 fue seleccionado y finalista de Young Architects Program 2012 (YAP_CONSTRUCTO) y obtuvo el primer lugar (junto a Nicolás Urzúa y José Tomás Rodríguez) en el concurso internacional "Ex Cárcel de Talca".

Actualmente ejerce asociado a Nicolás Urzúa, es profesor instructor de Taller de proyectos en la Escuela de Arquitectura de la Uc, editor de contenidos del sitio Plataforma Arquitectura y editor a cargo de la sección de arquitectura de la revista ED.
Nicolás Urzúa | Ayudante, Pontificia Universidad Católica de Chile, Santiago

Arquitecto, Pontificia Universidad Católica de Chile, 2011 y estudios de arquitectura, École Nationale Supérieure d'Architecture de Paris-Malaquais, 2008. Es socio del Colectivo MURO - a cargo de las propuestas de reconstrucción para el pueblo de Pumanque tras el terremoto de 2010cuyo trabajo fue expuesto en la XII Bienal de Arquitectura de Venecia. En 2012 fue seleccionado y finalista de Young Architects Program 2012 (YAP_CONSTRUCTO) y obtuvo el primer lugar (junto a Guillermo Hevia y José Tomás Rodríguez) en el concurso internacional "Ex Cárcel de Talca".

Actualmente ejerce asociado a Guillermo Hevia y es ayudante de Taller de proyectos en la Escuela de Arquitectura de la UC.

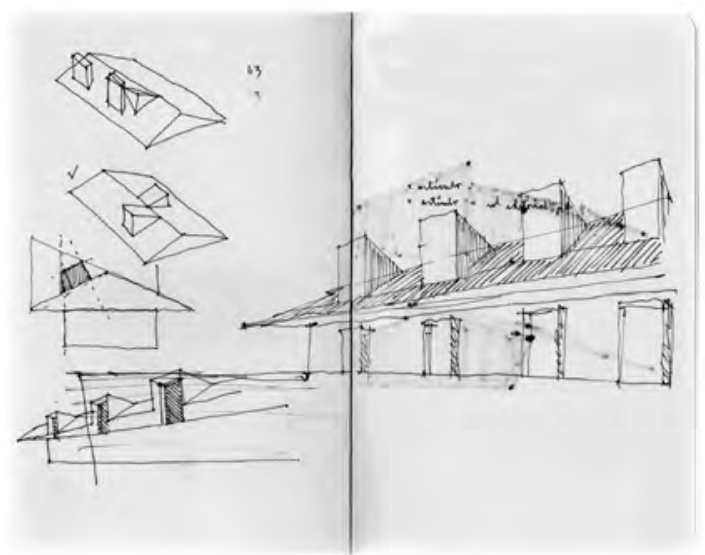




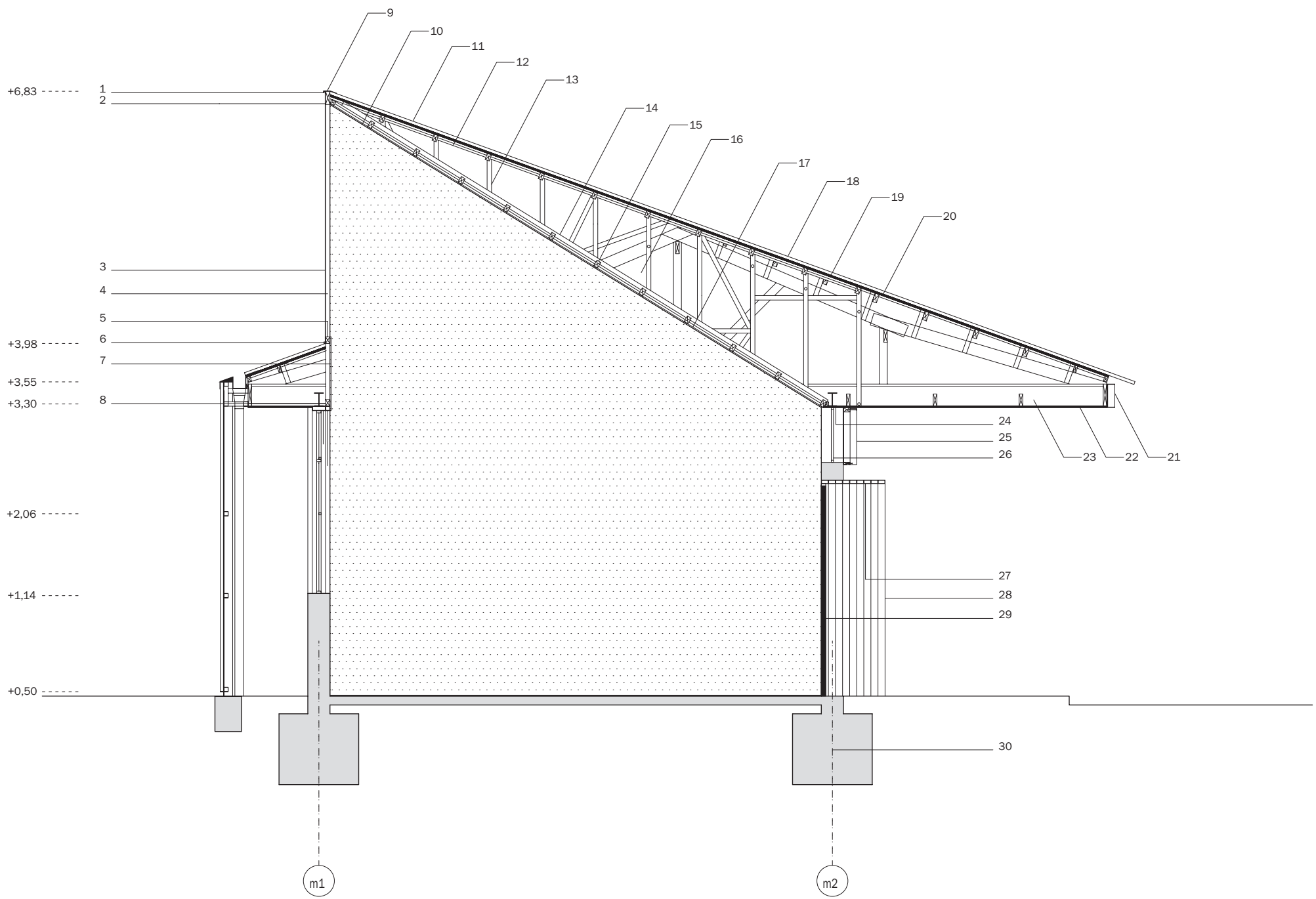

E. 1: 75

1. Barra Fe $100 \mathrm{~mm}$

2. Marco lucernario listón madera 6 × 2"

3. Vidrio laminado

4. Marco lucernario, listón madera $2 \times 3^{\prime \prime}$

5. Barra Fe $100 \mathrm{~mm}+$ ángulo metálico remate

6. Marco lucernario, listón madera $2 \times 3 "$

7. Yeso-cartón $12,5 \mathrm{~mm}$

8. Marco lucernario, listón madera 2 × 3"

9. Tapa cantos hojalatería

10. Yeso-cartón $12,5 \mathrm{~mm}$

11. Panel $\mathrm{e}=0,5 \mathrm{~m}$

12. Estructura madera superior $2 \times 3 "$

13. Estructura madera inferior Estructura madera $2 \times 3$

14. Anclaje cielo madera $1 \times 4 "$

15. Placa OSB inferior

16. Costanera madera $2 \times 3$

17. Aislación lana mineral

18. Panel $\mathrm{e}=0,5 \mathrm{~m}$
19. Fieltro

20. Placa OSB superio

21. Viga remate $\mathrm{Fe} C$ extruida $260 \times 80 \times 3 \mathrm{~mm}$

22. Cielo madera

23. Viga metálica cercha existente $150 \times 100 \times 5 \mathrm{~mm}$

24. Viga metálica cercha existente $250 \times 150 \times 5 \mathrm{~mm}$

25. Celosía madera $1 \times 3 "$

26. Ventana existente

27. Marco tubular superior puerta Fe $80 \times 40 \mathrm{~mm}$

28. Marco tubular lateral puerta Fe $80 \times 40 \mathrm{~mm}$

29. Puerta existente

30. Fundación existente
El replanteo de la condición constructiva y espacial de estas aulas propone fundamentalmente dos niveles de intervención a escala edilicia: una nueva envolvente traslúcida, perforada de acuerdo a diseño, y la construcción de lucernarios que proveen al interior de luz y altura. Palabras clave: Arquitectura - Chile, arquitectura educacional, lucernario, metal perforado.

A new take on the spatial and material conditions of these classrooms introduces two levels of intervention within the building scale: a new translucent, custom-perforated envelope, and skylights that provide light and higher interior heights.

Keywords: Architecture - Chile, educational buildings, skylight, perforated metal sheets. 


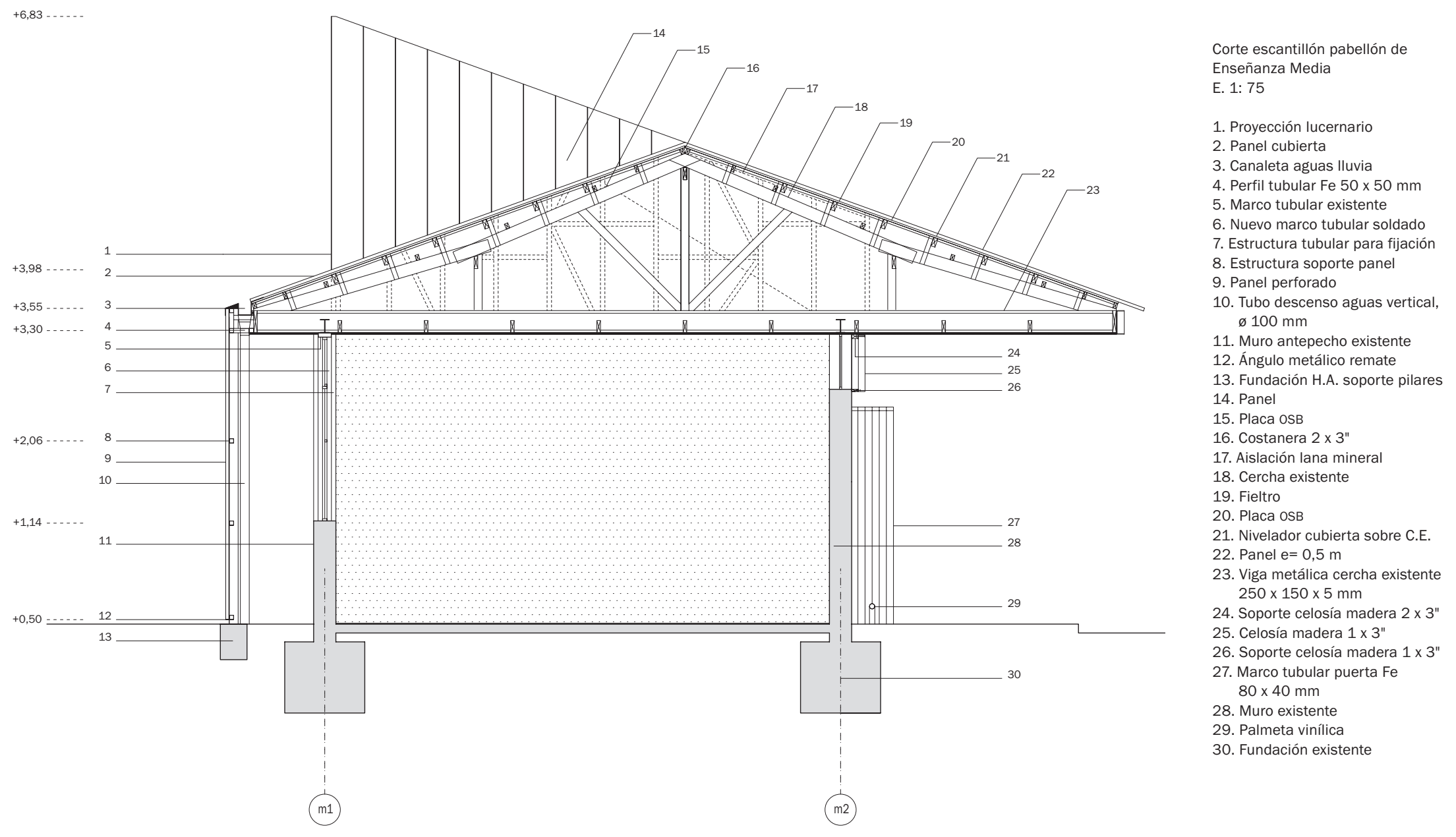

El proyecto aborda la renovación de las instalaciones de un colegio y la construcción de espacios para nuevos programas. La iniciativa está relacionada a la modernización de la infraestructura y a la renovación de la imagen e identidad institucional. Una serie de pabellones con cubiertas a dos aguas y patios interiores caracterizaba las instalaciones existentes; otras condiciones memorables eran la altura interior de los recintos y la generosidad de los aleros.

Por tratarse de una renovación en un establecimiento educacional en pleno funcionamiento, todos los trabajos de la construcción se debieron llevar a cabo durante dos meses del verano, correspondientes al periodo de vacaciones. Este es un dato fundamental para el proyecto, ya que la eficiencia (en la construcción y el montaje de diferentes elementos constructivos) fue requisito definitivo para el diseño de la propuesta. Para economizar costos y tiempos de trabajo se tomó la decisión de trabajar sólo con estructuras metálicas montadas en seco, mientras que los diferentes cerramientos se produjeron en serie en maestranzas, de modo de reducir la construcción al ensamble de piezas de similar formato.

Tres escalas de acción, vinculadas cada una a diferentes elementos, se encuentran en el proyecto.

La primera busca dar un orden al colegio en función de los cuatro patios existentes, transformándolos en cuatro momentos fundamentales y distintivos. Serán cuatro espacios con diferentes atmósferas, de acuerdo a sus usuarios y a las actividades que ellos realizan: juegos para los niños, cancha de deportes para el primer ciclo, espacios de conversación para el segundo ciclo y una plaza
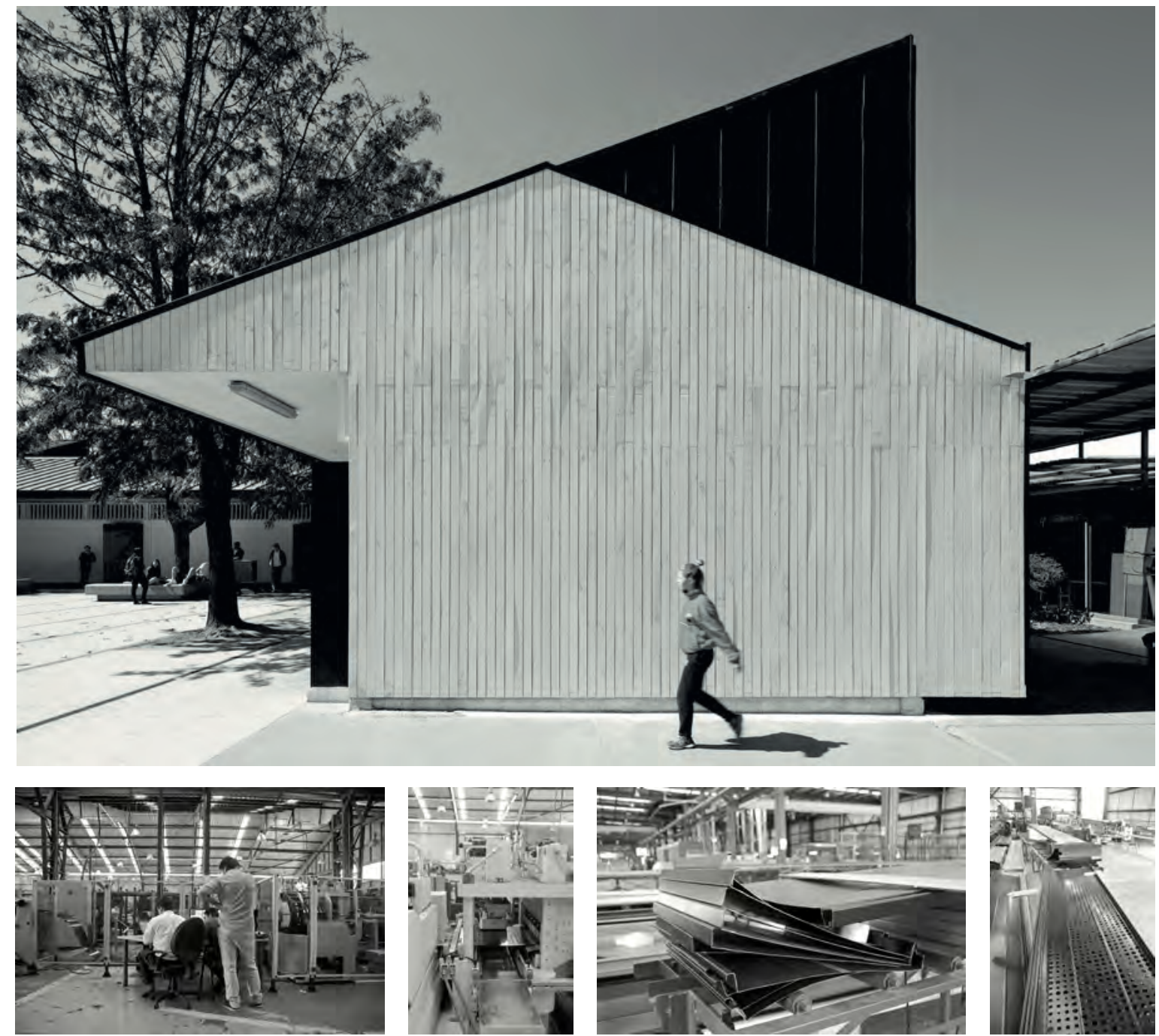
abierta para el tercer ciclo. 

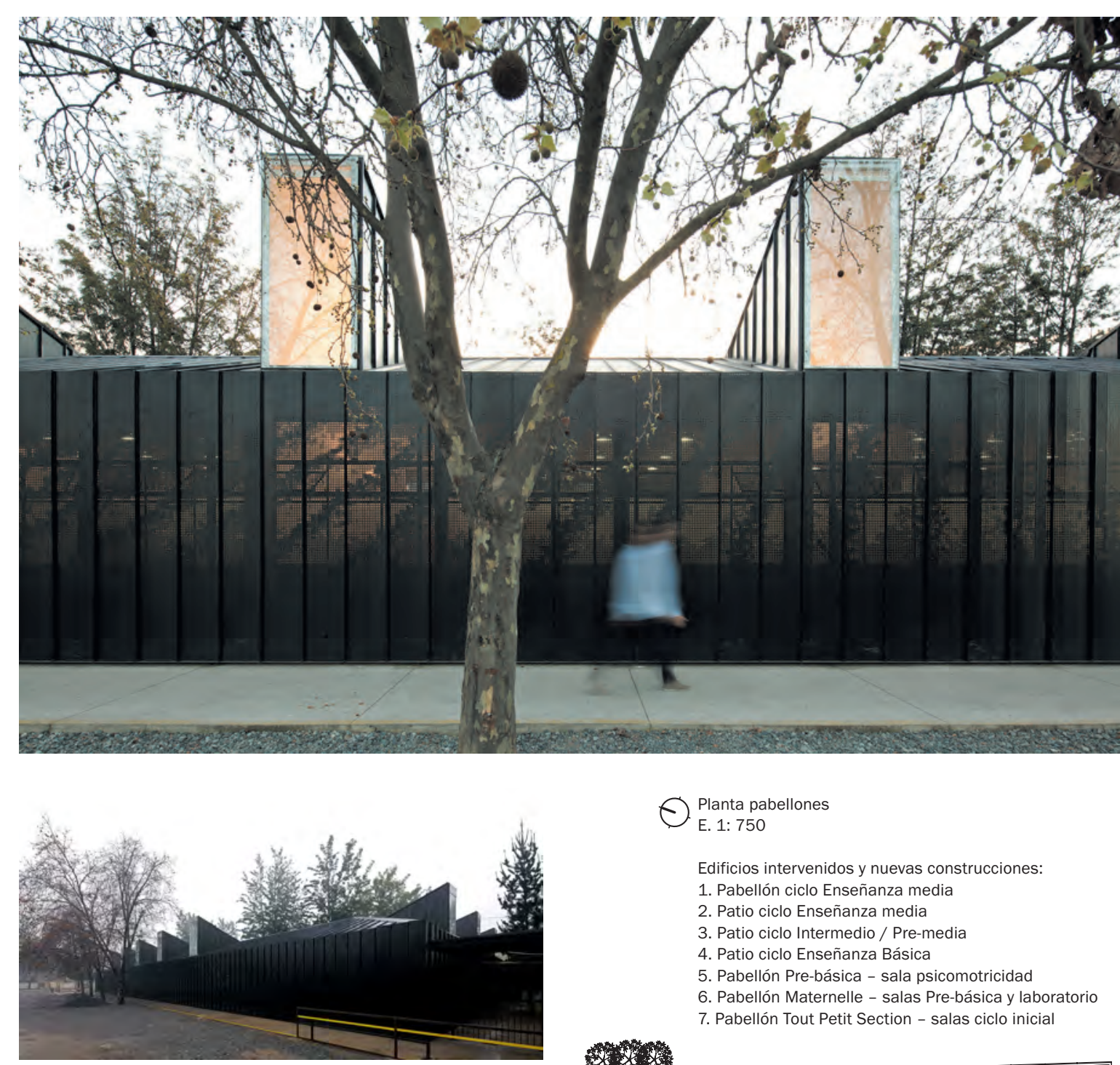

- Planta pabellones

Edificios intervenidos y nuevas construcciones: 1. Pabellón ciclo Enseñanza media

2. Patio ciclo Enseñanza media

3. Patio ciclo Intermedio / Pre-media

4. Patio ciclo Enseñanza Básica

5. Pabellón Pre-básica - sala psicomotricidad

6. Pabellón Maternelle - salas Pre-básica y laboratorio

7. Pabellón Tout Petit Section - salas ciclo inicial
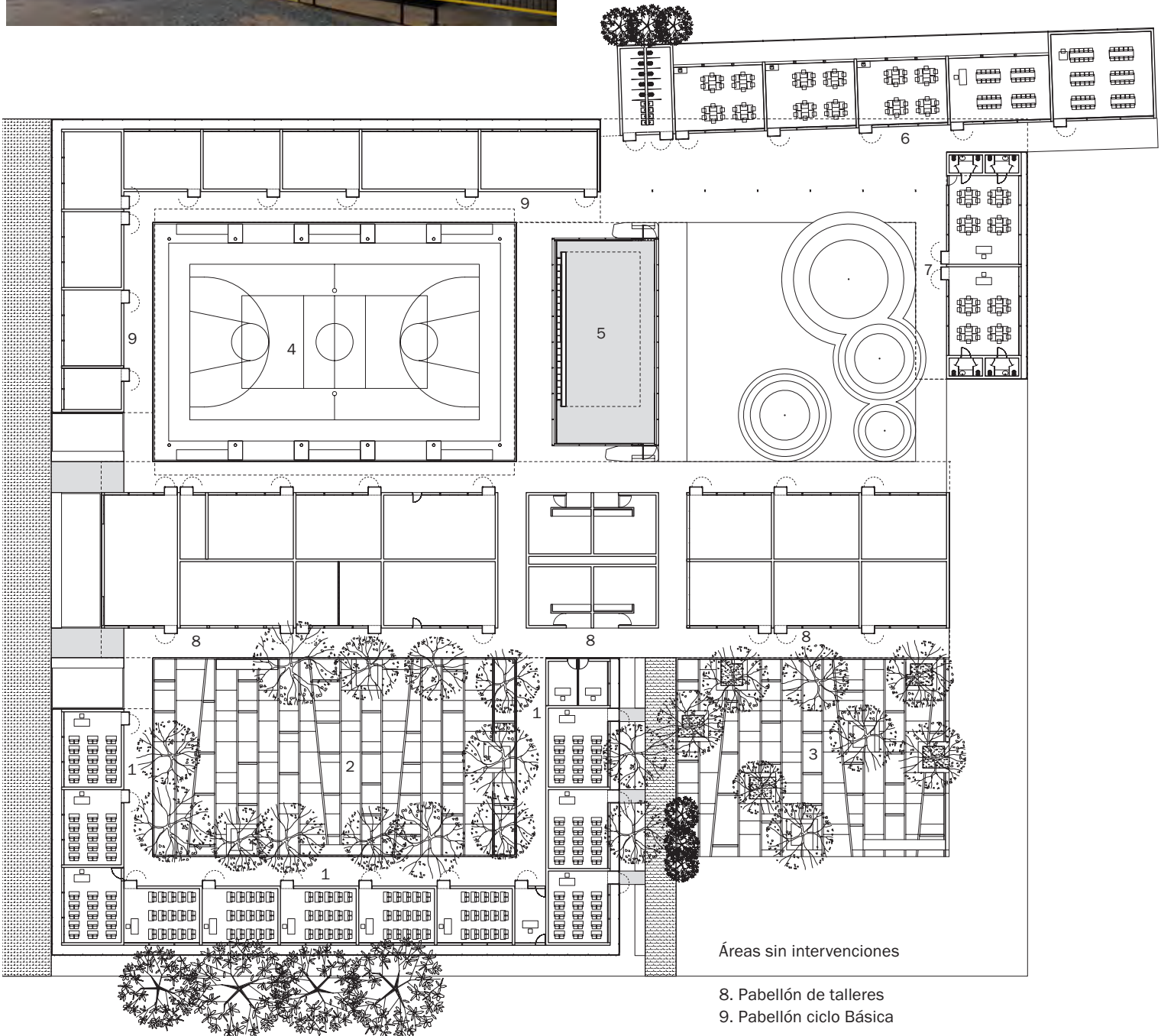

Areas sin intervenciones

8. Pabellón de talleres 9. Pabellón ciclo Básica
La segunda escala dice relación con los pabellones propiamente tales y se aborda empleando dos elementos constructivos. El primero es una nueva envolvente en base a placas continuas de metal perforado producidas industrialmente, las que permiten un montaje rápido y en seco. El diseño de cada paño de esta nueva fachada se vincula a diferentes motivos franceses, como viñedos o campos de lavanda, cuyas imágenes se transfieren a los paneles por medio de perforaciones realizadas según diseño. La envolvente metálica condiciona las vistas al exterior desde las salas de clases y al mismo tiempo aparece, retro iluminada, durante la tarde y la noche, definiendo y diferenciando cada fachada. El segundo elemento es un lucernario tipo, que se instaló en serie sobre las cubiertas. Ellos transforman la espacialidad interior de las salas, otorgándoles luz y ventilación natural, además de generar una nueva imagen para el colegio.

Una tercera escala vincula el proyecto y el cuerpo humano en proximidad. Esta relación se aborda por medio de un nuevo sistema de acceso a las aulas -que incorpora colores y gráficas en cada puerta-, un orden de celosías instalado delante de las ventanas para generar privacidad en las salas sin perder vistas ni luminosidad y por último nuevos pavimentos de colores que diferencian los interiores y les dan una cualidad ambiental.

La nueva construcción que incorpora la propuesta es un Pabellón de Psicomotricidad, un espacio libre y fluido para que los niños realicen actividades y juegos en un lugar interior. El pabellón está caracterizado por su estructura: se trata de una viga puente habitable, de rápido montaje y que se abre hacia el patio de juegos. Sobre el pabellón se descuelga una viga superior pensada como el soporte de columpios. Su interior se configura mediante una envolvente de madera que alberga un mueble para el almacenaje de los diferentes elementos de la sala y que genera, al mismo tiempo, una gran banca en toda su extensión. Este elemento incorpora una nueva escala para los niños.

El proyecto se construyó en 75 días. ARQ

\section{Bibliografía sugerida}

BROWNE, Tomás. "Exteriores de los edificios educacionales" Revista ARQ N 56 - Educación. Ediciones ARQ, Santiago, 2004. IRARRÁZAVAL, Sebastián. "Escuela modular". Revista ARQ No 77 - Urgencia y materia. Ediciones ARQ, Santiago, 2011. VILLALóN, Malva. “La educación en el cambio de siglo". Revista $A R Q N^{\circ} 56$ - Educación. Ediciones ARQ, Santiago, 2004.

COLEGIO ALIANZA FRANCESA JEAN MERMOZ | Arquitectos: Guillermo Hevia García, Nicolás Urzúa Soler | Ubicación: Curicó, Chile | Cliente: Corporación Educacional Alianza Francesa | Cálculo estructural: ALPA Ingeniería - Alfonso Pacheco | Construcción: Luis Farías | Proyecto eléctrico: Luis Farías | Proyecto sanitario: Luis Farías | Materialidad: estructura de muros de albañilería, estructura metálica y cerchas de madera; cerramientos en panel perforado Hunter Douglas CD 500SL, cristal laminado de $8 \mathrm{~mm}$; cubiertas en panel perforado Hunter Douglas CD 500sL; pavimentos interiores vinílicos y tabiquerías en madera de pino cepillada $1 \mathrm{x}$ 3 " y paneles de yeso cartón pintados con esmalte al agua Presupuesto: US\$ 290/m²; UF $6 / \mathrm{m}^{2}$ (renovación); US\$ 590/ $\mathrm{m}^{2}$; UF $12,3 / \mathrm{m}^{2}$ (nuevo pabellón) | Superficie de terreno: $34.800 \mathrm{~m}^{2}$ | Superficie construida: $1.450 \mathrm{~m}^{2}$ (renovación), $210 \mathrm{~m}^{2}$ (nuevo pabellón) | Año de proyecto: 2011 | Año de construcción: 2012 | Fotografía: Nicolás Saieh. 